\title{
Case Report: \\ An 8-year-old Boy With Three Failed Suicide Attempts by Hanging: A Case Report
}

\author{
Ali Ashraf Mozafari ${ }^{1} \odot$, Amir Adibi ${ }^{2} \odot$, Elham Shafiei ${ }^{1} \odot$, $^{*}$ Ali Sahebi ${ }^{1} \odot$
}

1. Non-Communicable Diseases Research Center, Ilam University of Medical Sciences, Ilam, Iran.

2. Department of Child and Adolescent Psychiatry, Faculty of Medicine, Ilam University of Medical Sciences, Ilam, Iran.

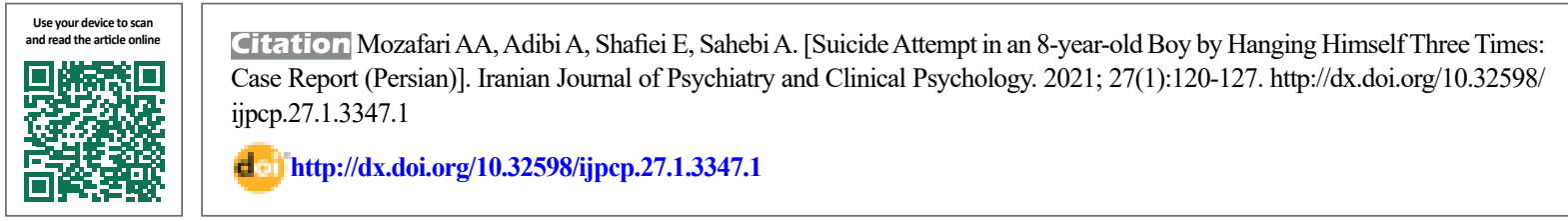

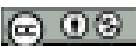

Received: 14 Feb 2020 Accepted: 02 Aug 2020 Available Online: 01 Apr 2021

Key words:

Suicide attempt, Major depressive disorder, Child behavior, Child neglect

\section{A B STRACT}

Objectives Suicidal behavior in children under 10 years of age is a warning and a matter of concern. It can be an important indicator for evaluating the mental health of individuals in the community. Case Report This study reported an 8-year-old boy who had three failed suicide attempts by hanging. The psychiatric assessment revealed that he had major depressive disorder which had not been diagnosed. Conclusion Evaluation of his behavior by using the Child Behavior Checklist completed by his parents showed that he had the highest scores in aggressive behavior, attention problems, rule-breaking behavior, and anxious/depressed subscales. Lack of parental attention to meeting the needs and creating a supportive environment may have led to behavioral-emotional disorders and risky behaviors such as suicide in the boy.

\section{Extended Abstract}

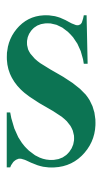

\section{Introduction}

uicide attempt in children under 10 years of age is a warning and a matter of concern. It can be an important indicator for evaluating the mental health of individuals in the community. Although all age groups are affected by this phenomenon, the highest incidence is related to 15-30 years age group. Studies on suicidal behavior in children are limited and with small sample sizes. Therefore, its global epidemiology and risk factors in the age group $<10$ years are unknown and there is need for identifying the risk factors contributed to suicidal behavior in children. The Centers for Disease Control and Prevention reported a suicide rate of 0.17 per
100000 people in youth between the ages of 5 and 11 years. Between 2008 and 2015, a total of 14,852 children aged 5 -11 years with suicidal ideation or suicide attempt were hospitalized in the United States, mostly due to mood disorders including suicide. During 1993-2001 in Iran, there was a total of 3225 suicides among children and adolescents in Iran where the ratio of boys to girls was 3.7:1.

\section{Case Report}

The case was an 8-year-old boy with three suicide attempts by hanging referred to the psychiatric clinic of Shahid Mostafa Khomeini Hospital in Ilam, Iran by his parents. They were living in one of the villages of Ilam city. This family had five children; the case was the fourth child of this family studying in the grade-2 elementary school. He had aver-

* Corresponding Author:

Ali Sahebi

Address: Non-Communicable Diseases Research Center, Ilam University of Medical Sciences, llam, Iran.

Tel: +98 (918) 3434703

E-mail: ali.sahebi.phd@gmail.com 
age grades with no hyperactivity or aggressive behaviors at school. His parents stated that he hanged himself in a barn three times during 2018-2019, but he had been saved by his older sister. His parents had elementary education with a monthly family income of 1-1.5 million Tomans. They had no family history of suicide or mental illness. Rural life and large family size, cause young children not receive much psychological and emotional support. Psychiatric assessment was performed in a clinic. The results revealed that he had a major depressive disorder (undiagnosed). Hence, he treated with $12.5 \mathrm{mg}$ sertraline daily for one week and then the dosage increased to $25 \mathrm{mg}$ daily. During this period, psychotherapy based on emotional release was carried out. Throughout this period, which lasted several weeks, his responds to treatment somewhat improved such that in the fifth session, he began to cry. Then, his behavior was assessed by parents using the Child Behavior Checklist (CBCL). He is currently under treatment by a psychiatrist and is visited monthly, and has not attempted suicide again.

\section{Discussion and Conclusion}

This study aimed to report behavioral-emotional disorders in an 8-year-old boy with attempted suicide by using the CBCL and psychiatric diagnosis. The CBCL was completed by one of his parents. It can be completed as a self-report or through interview. It measures 8 emotional-behavioral problems under three categories of internalized problems, externalized problems, and general problems. The CBCL scores showed that the boy had higher scores in aggressive behavior, attention problems, rule-breaking behavior, and anxious/depressed subscales. The externalized problems were predominant. His psychiatric assessment revealed that he had major depressive disorder. Suicide attempt among children aged $<10$ years compared to other age groups is rare, and are commonly had specific behavioral disorders. A stressful event may trigger suicide in children who have mental problem such as depression. These children are usually anxious and inactive, and change their behaviors quickly. Untreated depression is an important factor in suicide attempts in children. In the study boy, undiagnosed depression and lack of support by parents led to suicide attempts. Parent-child conflict is a hidden factor that accelerates suicide attempts among children, and hanging is one of the most common method of suicide in these children.

The boy had reasonable demands such as being loved, and buying him toys and clothes but his parents could not provide them due to high number of children in the family and the parents' financial inability. These factors cause the inability of parents to meet the various needs of children and creates child neglect in the family. Children with depression have a high tendency to commit suicide. Exposure to acute stressors can exacerbate this condition. Therefore, improving the parent-child relationship and eliminating the children's emotional deprivation can be effective in reducing the incidence of suicide in children. Emotionalbehavioral problems in childhood are associated with many functional impairments in various areas of life. Therefore, preventive services in the health care system such as a safe care program as in-home training program with an emphasis on child-parent conflicts, safe family environment, and child health can effectively reduce these disorders. Moreover, teachers need to be educated on the mental health to detect students' behavioral disorders.

\section{Ethical Considerations}

\section{Compliance with ethical guidelines}

This article is taken from the registered cases of suicide attempts in Ilam province approved by Ilam University of Medical Sciences (Ethics Code: IR.MEDILAM.REC.1398.056).

\section{Funding}

This research did not receive any grant from funding agencies in the public, commercial, or non-profit sectors.

\section{Authors contributions}

Conceptualization and supervision: Amir Adibi; Research and data collection: Ali Ashraf Mozafari and Ali Sahebi; Writing - original draft, and writing - review \& editing: Elham Shafiei; Review and editing: Ali Sahebi and Ali Ashraf Mozafari.

\section{Conflicts of interest}

The authors declared no conflict of interest.

\section{Acknowledgements}

We would like to thanks the Clinical Research Development Unit, Shahid Mostafa Khomeini Hospital, Ilam University of Medical Sciences. 
This Page Intentionally Left Blank 
اقدام به خودكشى در يك يسربجه 1ساله با سهبار سابقه حلق آويزّ: تَزارش موردى

\author{
على اشرف مظفرى' ه، امير اديبى' ه، الهام شفيعى' ه "على صاحبى' \\ 1. ا.مركز تحقيقات بيمارى هاى غيرواكير، دانشكاه علوميزشكى ايلامه ايلامه ايران.

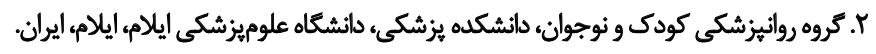

\section{حكيد}

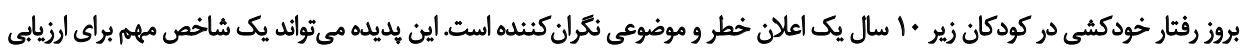

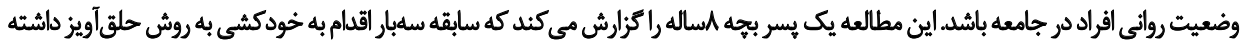

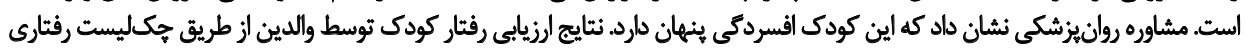

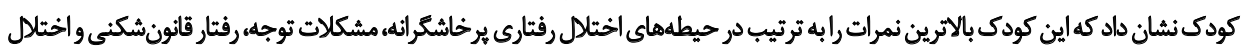

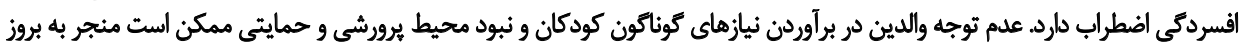

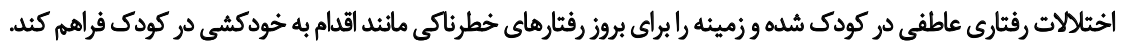

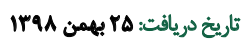

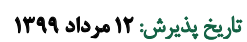

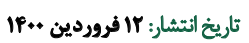

: Lojlgen

اقدام به خودكشي، اختلال افسردكى اساسي، رفتئار

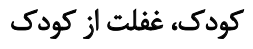

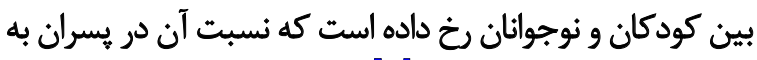

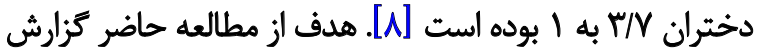

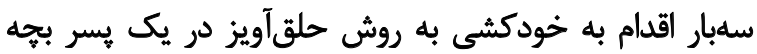

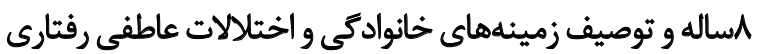
در كودى و ارتباط آن با اقدام به خودكشى خدوانيف است.

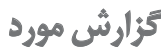

بيمار يك بسر بهّه مساله است كه والدين با شكايت اقدام

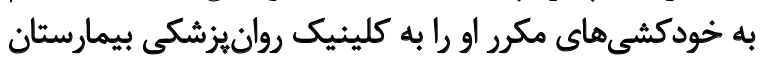

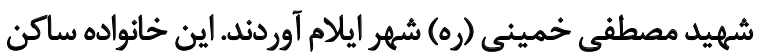

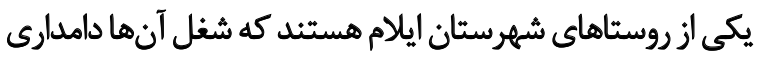

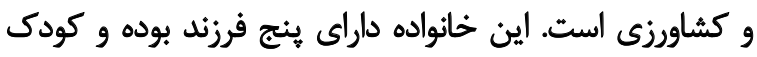

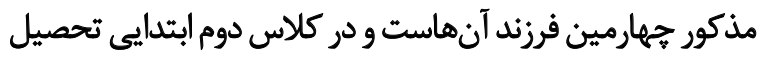

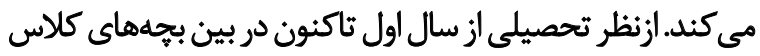

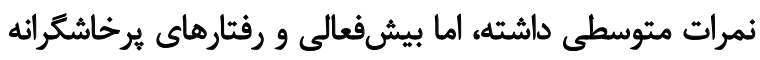

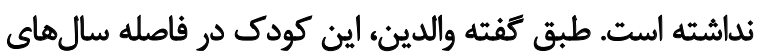

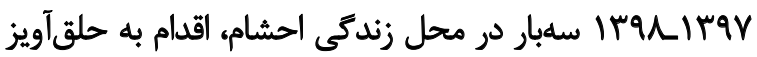

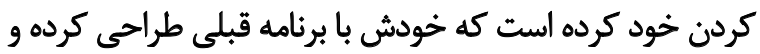

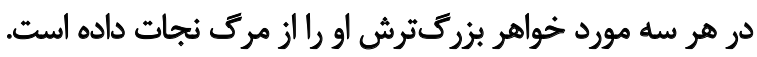

مقدمه

اقدام به خودكشى يكى از مبهمترين شاخصهاى بهدئ

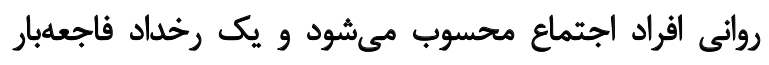

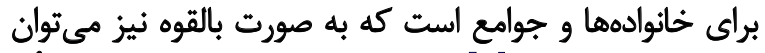

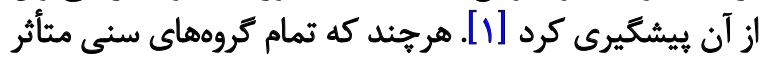

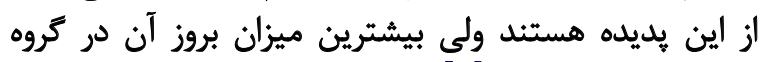

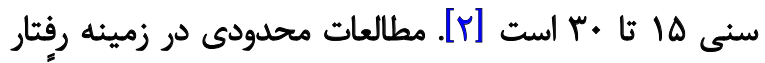

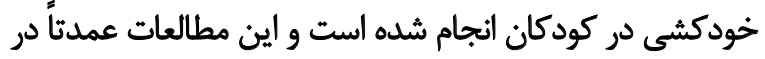

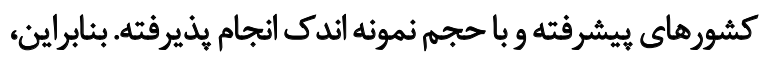

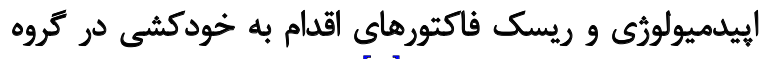

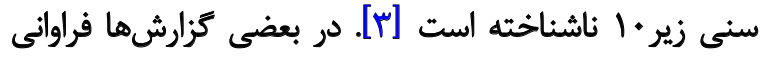

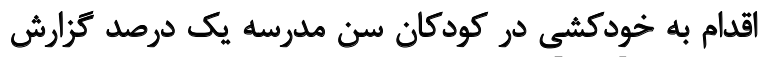

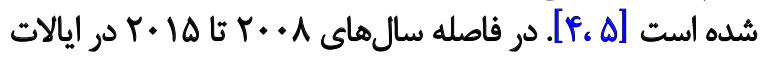

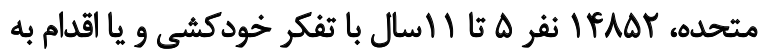

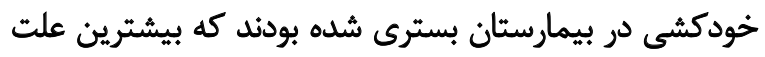

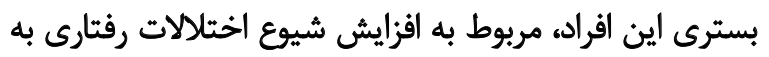

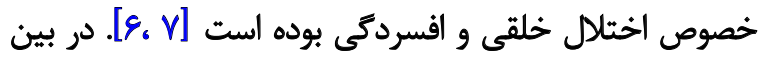

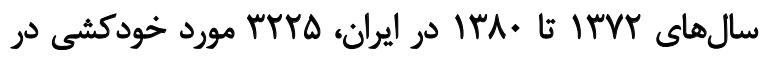

\title{
: نويسئده مسئول:
}

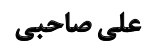

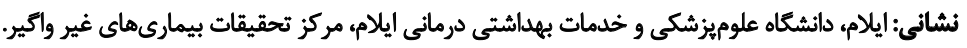

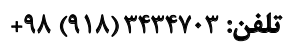
ali.sahebi.phd@gmail.com بلكث الكترونيكي: 
تجزيه و تحليل يرسشنامه CBCL نشان داد كه اين كودى

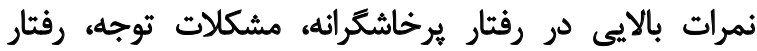

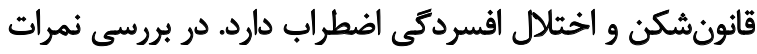

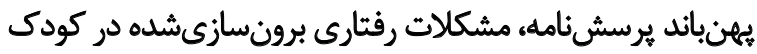

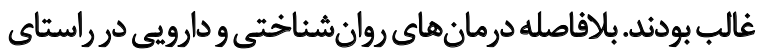

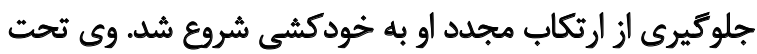

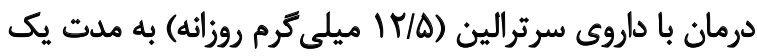

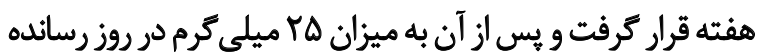

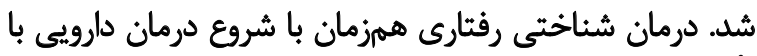

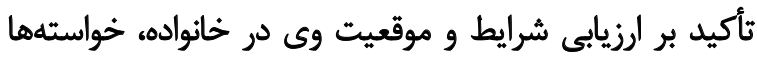

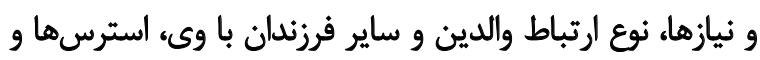

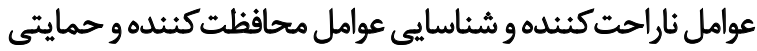

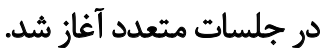

در طول جلسات از تكنيك كوش دادن فعال استفاده شد تا

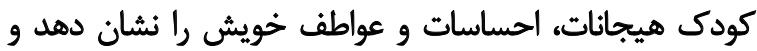

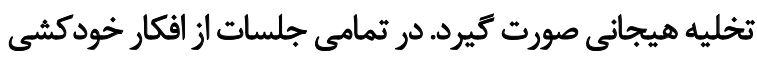

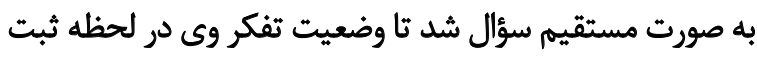

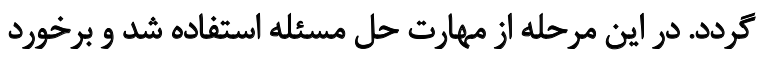

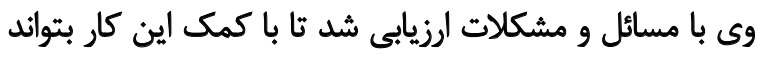

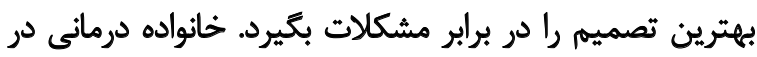

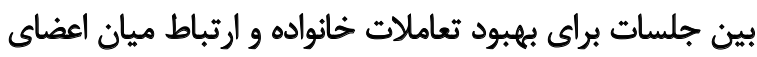

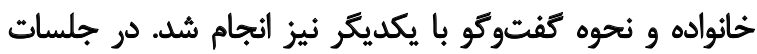

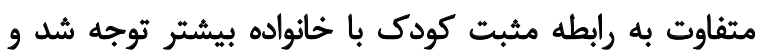

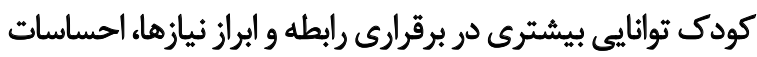

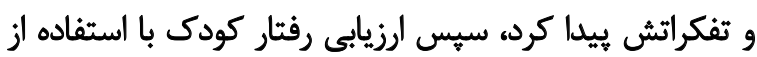

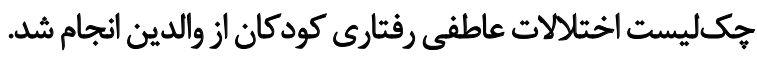

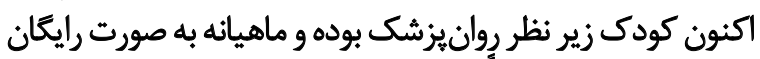

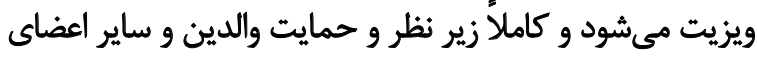

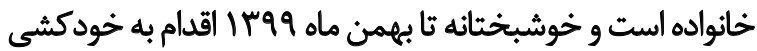

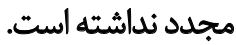

$\stackrel{\leftrightarrow}{\oplus}$

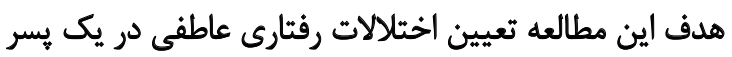

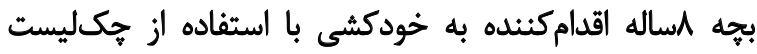

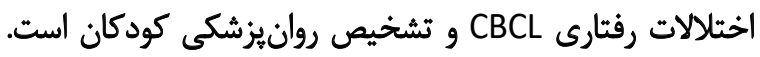

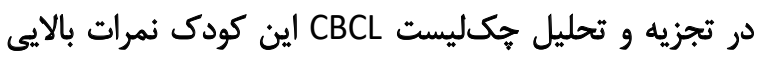

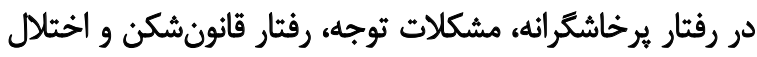

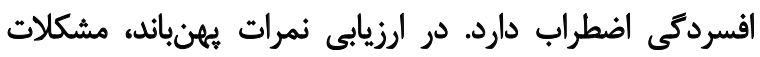

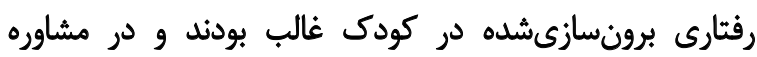

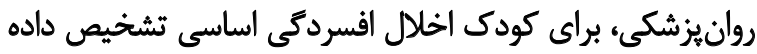

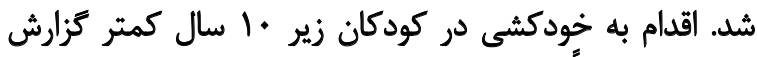

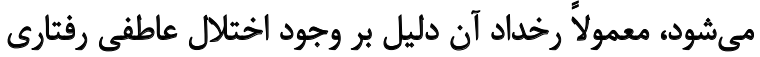

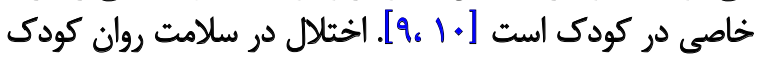

اقدام به خودكشى اول در دى ماه لوجسا، دومى در فروردين

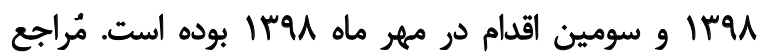

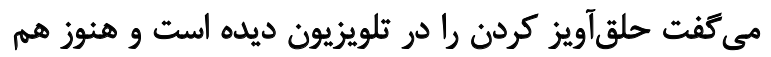

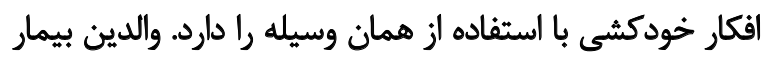

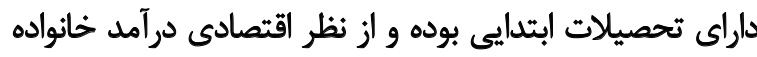

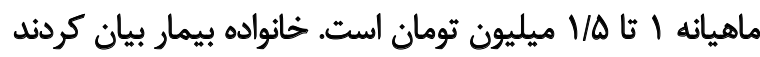

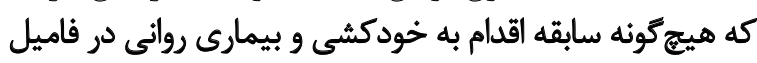

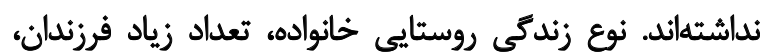

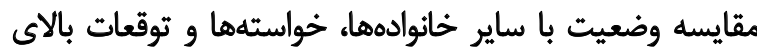

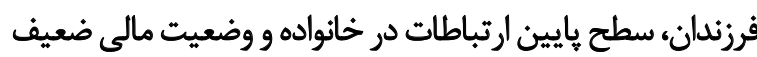

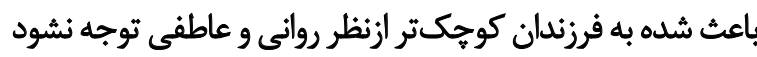

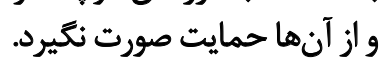

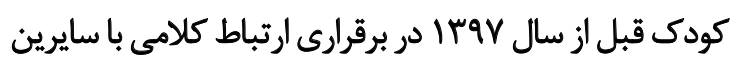

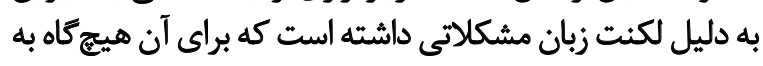

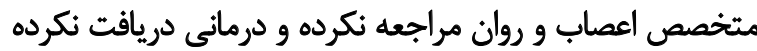

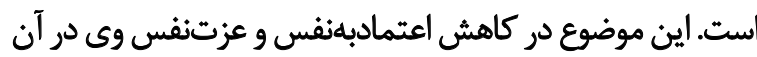

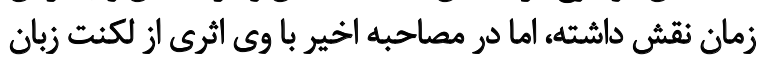

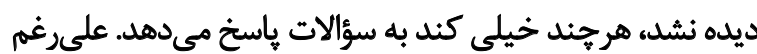

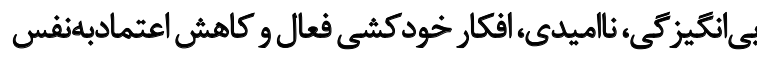

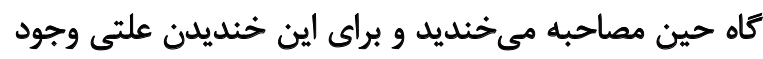

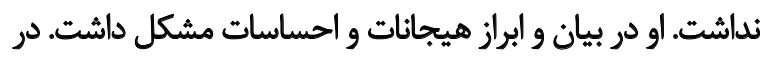

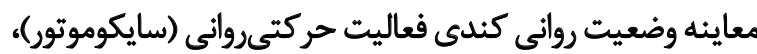

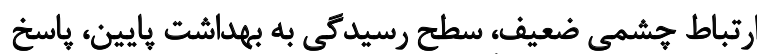

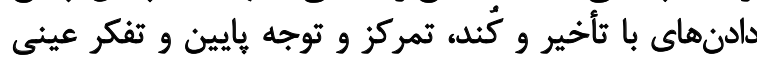

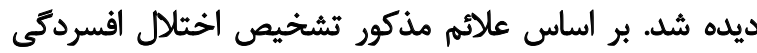

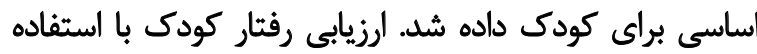

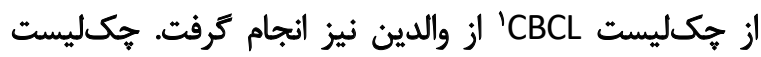

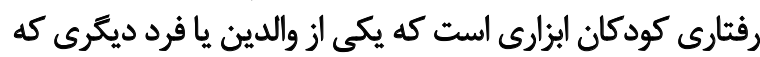

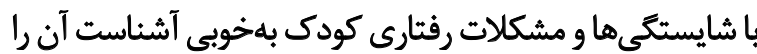

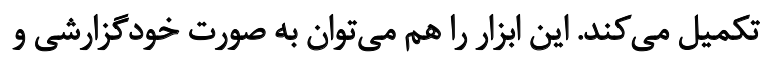
هم به صورت مصاحبه اجرا كرد.

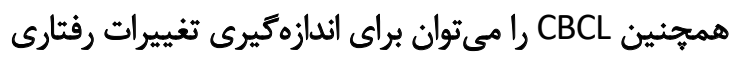

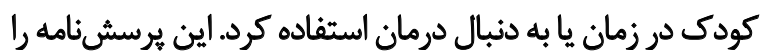

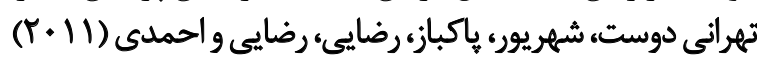

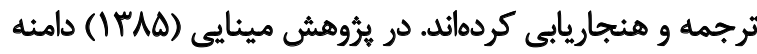

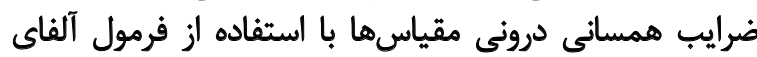

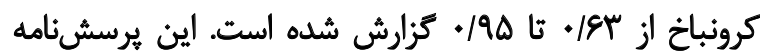

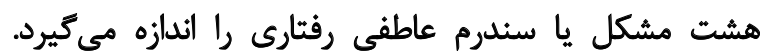

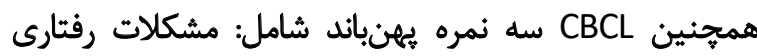

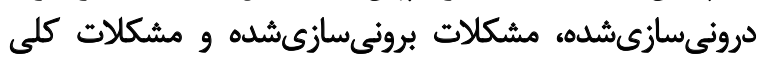

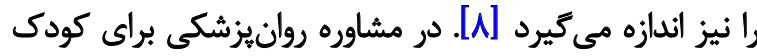
اخلال افسردكى اساسى تشخيص داده شد.

1. Child Behavior Checklist 
از آنجايي كه محل سكونت كودى در روستا بوده و دسترسى سي

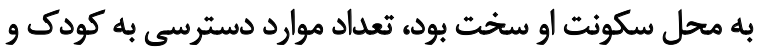
ساير اعضاى خانواده آن به مراتب كمتر مى بود.

با توجه به اينكه بروز افسردكى در سنين كودكى نيز وجود

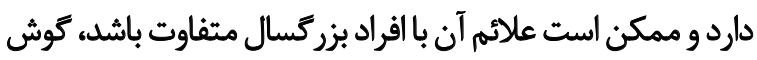

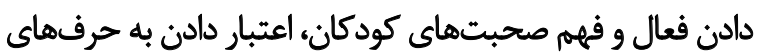

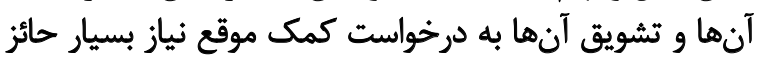

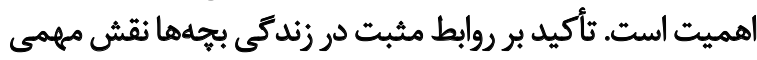
در ارتقا سلامت روان آنها خواهد داشت داشت.

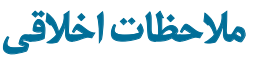 \\ يبروى از اصول اخلاق يُوهشش}

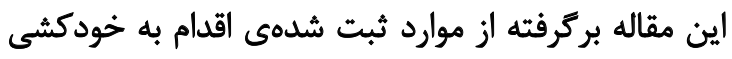
استان ايلام مصوب دانشكاه علوميزشكى ايلام است (كد اخديت اقداق:

. IR.MEDILAM.REC.1398.056

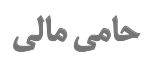

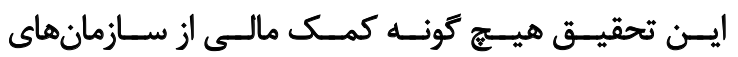

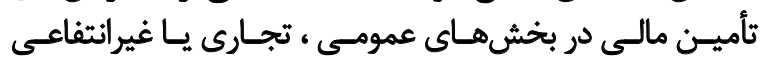

$$
\text { دريافت نكـرد. }
$$

$$
\text { مشاركت ثويسند مان }
$$

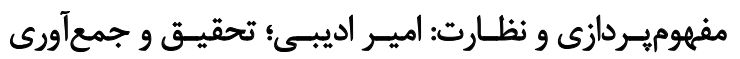

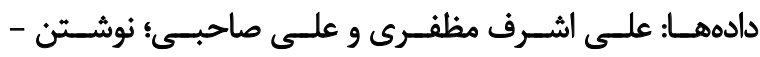

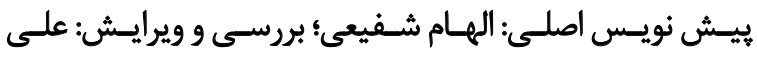

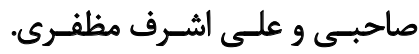

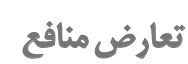

طبق اعلام نويسندكان اين مطالعه تعارض منافع ندارد.

$$
\text { تقديو وتشكر }
$$

بدينوسـيله از واحــد توسـعه تحقيقـات بالينـى بيمارســان

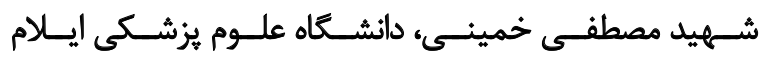
تشـكر مينمائيـمه خمين.

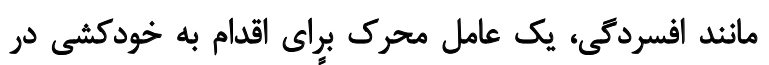

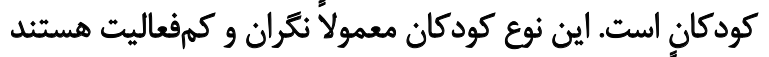

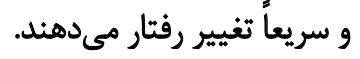

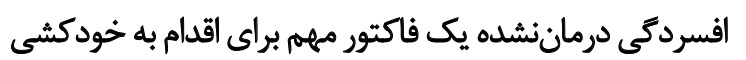

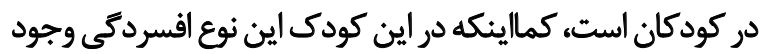

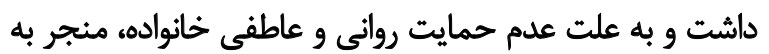

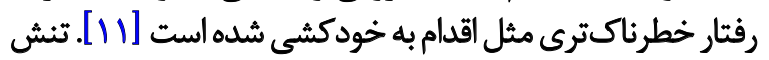

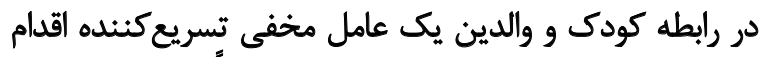

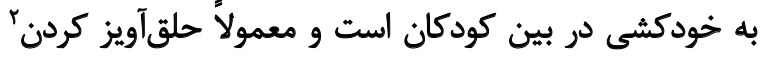

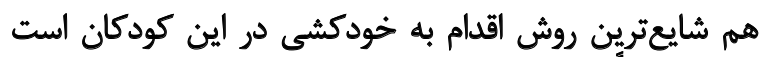

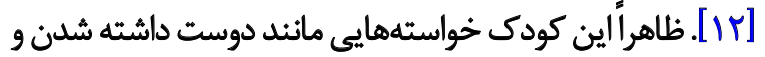
داشتن اسباب بازى و لباس از والدين داشته است كائ كه نتواندانستهانداند

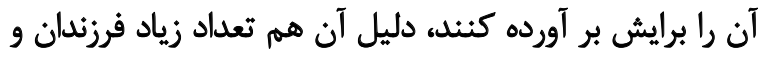

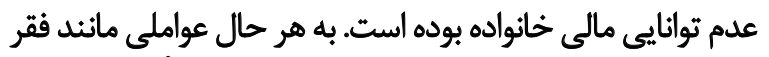

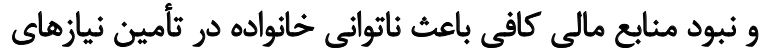

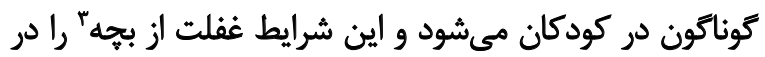

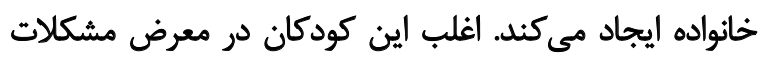

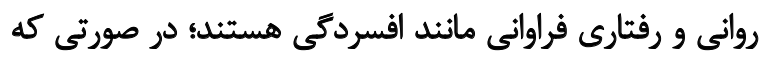

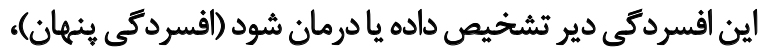

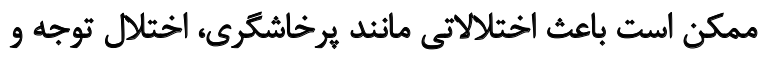

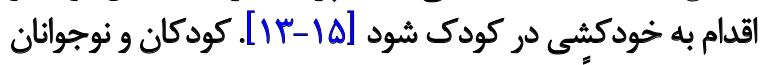

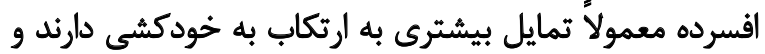

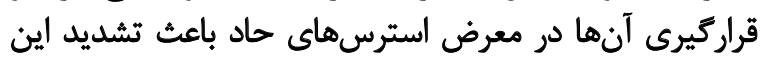

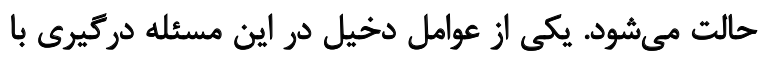

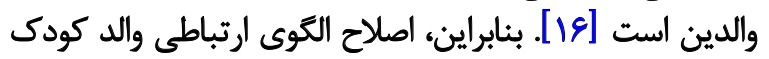

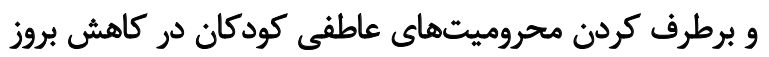

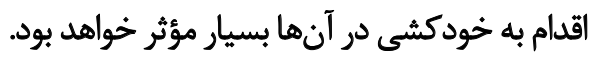

اختلالات رفتارى عاطفى در دوران كودكى بان آسيبهاي

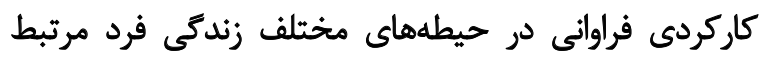

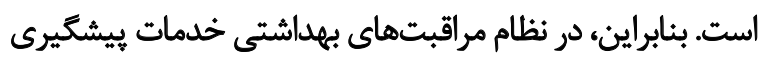

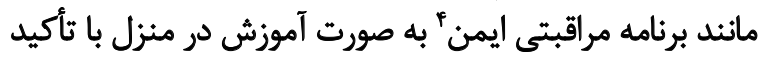

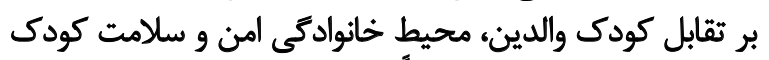

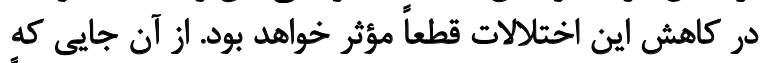

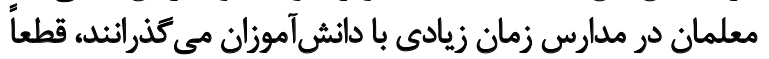

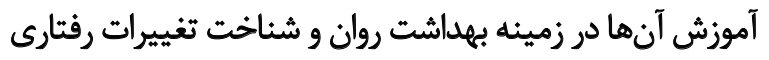

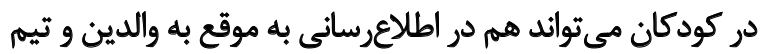

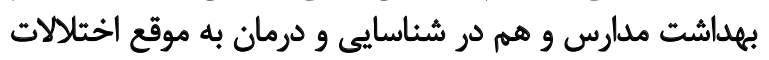

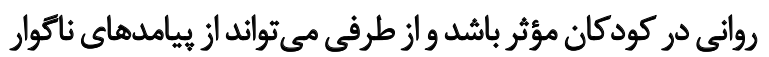

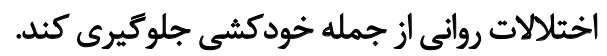




\section{References}

[1] Zainum K, Cohen MC. Suicide patterns in children and adolescents: A review from a pediatric institution in England. Forensic Science, Medicine, and Pathology. 2017; 13(2):115-22. [DOI:10.1007/s12024-017-9860-y] [PMID]

[2] Sinyor M, Tse R, Pirkis J. Global trends in suicide epidemiology. Current Opinion in Psychiatry. 2017; 30(1):1-6.[DOI:10.1097/ YCO.0000000000000296] [PMID]

[3] Bridge JA, Asti L, Horowitz LM, Greenhouse JB, Fontanella CA, Sheftall AH, et al. Suicide trends among elementary school-aged children in the United States from 1993 to 2012. JAMA Pediatrics. 2015; 169(7):673-7. [DOI:10.1001/jamapediatrics.2015.0465] [PMID]

[4] Sheftall AH, Asti L, Horowitz LM, Felts A, Fontanella CA, Campo JV, et al. Suicide in elementary school-aged children and early adolescents. Pediatrics. 2016; 138(4):e20160436. [DOI:10.1542/ peds.2016-0436] [PMID] [PMCID]

[5] Pfeffer CR, Zuckerman S, Plutchik R, Mizruchi MS. Suicidal behavior in normal school children: A comparison with child psychiatric inpatients. Journal of the American Academy of Child Psychiatry. 1984; 23(4):416-23. [DOI:10.1016/S00027138(09)60319-8]

[6] Plemmons G, Hall M, Doupnik S, Gay J, Brown C, Browning W, Casey R, Freundlich K, et al. Hospitalization for suicide ideation or attempt: 2008-2015. Pediatrics. 2018; 141(6):e20172426. [DOI:10.1542/peds.2017-2426] [PMID]

[7] In-Albon T, Bürli M, Ruf C, Schmid M. Non-suicidal self-injury and emotion regulation: a review on facial emotion recognition and facial mimicry. Child and Adolescent Psychiatry and Mental Health. 2013; 7(1):1-1. [DOI:10.1186/1753-2000-7-5] [PMID] [PMCID]

[8] Tehrani-Doost M, Shahrivar Z, Pakbaz B, Rezaie A, Ahmadi F. Normative data and psychometric properties of the child behavior checklist and teacher rating form in an Iranian community sample. Iranian Journal of Pediatrics. 2011; 21(3):331-342. [PMCID] [PMID]

[9] Miotto P, De Coppi M, Frezza M, Petretto D, Masala C, Preti A. Suicidal ideation and aggressiveness in school-aged youths. Psychiatry Research. 2003; 120(3):247-55. [DOI:10.1016/S01651781(03)00193-8]

[10] Ghaffari M, Ahmadi A, Abedi MR, Fatehizade M, Baghban I. Impulsivity, substance abuse, and family/friends history of suicide attempts in university students with and without suicidal ideation. Iranian Journal of Psychiatry and Behavioral Sciences. 2011; 5(2):99-105. [PMCID] [PMID]

[11] Tishler CL, Reiss NS, Rhodes AR. Suicidal behavior in children younger than twelve: A diagnostic challenge for emergency department personnel. Academic Emergency Medicine. 2007; 14(9):810-8. [DOI:10.1197/j.aem.2007.05.014] [PMID]

[12] Soole R, Kõlves K, De Leo D. Suicide in children: A systematic review. Archives of Suicide Research. 2015; 19(3):285-304. [DOI:10 .1080/13811118.2014.996694] [PMID]

[13] Psychogiou L, Moberly NJ, Parry E, Nath S, Kallitsoglou A Russell G. Parental depressive symptoms, children's emotional and behavioural problems, and parents' expressed emotionCritical and positive comments. PloS One. 2017; 12(10):e0183546. [DOI:10.1371/journal.pone.0183546] [PMID] [PMCID]
[14] Barnett WS, Belfield CR. Early childhood development and social mobility. The Future of Children. 2006; 16(2):73-98. [DOI:10.1353/foc.2006.0011] [PMID]

[15] Rhodes AE, Boyle MH, Bethell J, Wekerle C, Goodman D, Tonmyr L, et al. Child maltreatment and onset of emergency department presentations for suicide-related behaviors. Child Abuse \& Neglect. 2012; 36(6):542-51. [DOI:10.1016/j.chiabu.2012.04.006] [PMID]

[16] Liu X, Gentzler AL, Tepper P, Kiss E, Kothencnè VO, Tamás Z, et al. Clinical features of depressed children and adolescents with various forms of suicidality. Journal of Clinical Psychiatry. 2006; 67(9):1442. https://www.psychiatrist.com/cart/ 
This Page Intentionally Left Blank 